Philosophie ANTIQUE
Philosophie antique

Problèmes, Renaissances, Usages

15 | 2015

Questions sur le scepticisme pyrrhonien

\title{
Philippe sOULIER, Simplicius et l'infini
}

\section{Claire Louguet}

\section{(2) OpenEdition \\ Journals}

Édition électronique

URL : https://journals.openedition.org/philosant/482

DOI : 10.4000/philosant.482

ISSN : 2648-2789

\section{Éditeur}

Éditions Vrin

\section{Édition imprimée}

Date de publication : 24 novembre 2015

Pagination : 291-294

ISBN : 978-2-7574-1141-4

ISSN : 1634-4561

\section{Référence électronique}

Claire Louguet, « Philippe soulıer, Simplicius et l'infini », Philosophie antique [En ligne], 15 | 2015, mis en ligne le 01 novembre 2018, consulté le 03 décembre 2022. URL : http://journals.openedition.org/ philosant/482 ; DOI : https://doi.org/10.4000/philosant.482

\section{(c) $(1) \odot$}

Creative Commons - Attribution - Pas d'Utilisation Commerciale - Pas de Modification 4.0 International - CC BY-NC-ND 4.0

https://creativecommons.org/licenses/by-nc-nd/4.0/ 
les individus. On voit donc que la pensée de Plotin affectionne les paradoxes. L'ouvrage d'A. Michalewski en présente et en affronte quelques-uns : l'Un peut donner parce qu'il ne possède pas ce qu'il donne, les Formes sont autarciques parce qu'elles dépendent du premier principe, l'individualité trouve son principe dans l'intelligible. Mais elle montre qu'ils peuvent se résoudre si l'on reconstitue la théorie originale de la production et de la puissance causale à l'intérieur de laquelle ils prennent place : la puissance des Formes intelligibles s'explique alors par un dynamisme qui leur est propre et dont elles héritent pourtant de leur terme supérieur. Ce qui peut apparaître contradictoire dans la pensée de Plotin est justement ce qui permet de penser la puissance causale de l'Intellect et finalement d'autres réalités à l'exclusion de la matière. Cette intuition, qui était déjà celle de Jean Trouillard dans la Procession plotinienne, trouve dans l'ouvrage d'A. Michalewski une nouvelle confirmation.

Sylvain Roux

Université de Poitiers

Philippe Soulier, Simplicius et Pinfini, Paris, Les Belles Lettres, 2014, « Anagôgê », 595 p. ISBN 978-2-251-42016-5

L'ouvrage, précédé d'une préface de $\mathrm{Ph}$. Hoffmann, reprend la première partie de la thèse que $\mathrm{Ph}$. Soulier a soutenue en 2010 . Il présente une étude très précise des enjeux philosophiques des pages que Simplicius consacre au commentaire des chapitres 4 à 8 du livre III de la Physique d'Aristote (In Physicam, 451-517 Diels). Il contient également une annexe présentant un résumé analytique du texte de Simplicius, une bibliographie, trois index (noms anciens, auteurs modernes, passages cités) et une table des matières très détaillée.

Dans la première partie («La thèse philosophique de Simplicius sur l'æ̋ $\pi \varepsilon l-$ pov », p. 25-73), après avoir présenté brièvement deux interprétations modernes de l'infini aristotélicien (Hintikka et Wieland), l'auteur en vient à celle de Simplicius, qu'il qualifie de « réaliste ». Il soutient que, en réduisant le concept aristotélicien de « pensée » (vónoı, Physique, III 4 et 8 ) à une « représentation » $(\dot{\varepsilon} \pi i v \circ \alpha)$ ou « imagination » $(\phi \alpha \nu \tau \alpha \sigma i \alpha)$, Simplicius procède à une « démystification conceptuelle » qui permet de mieux mettre en évidence la « réalité du procès à l'infini ». Soulier voit dans l'interprétation de la vónбıs en termes de $\phi \alpha \nu$ $\tau \alpha \sigma i \alpha$ ou d'ż $\pi$ ivoı $\alpha$ un geste fort ; cependant on pourrait se demander si Simplicius ne se contente pas d'expliciter la pensée d'Aristote (l'exemple d'Aristote consistant à penser un homme faisant plusieurs fois sa taille ou excédant la taille de la ville, Phys. III 8, 208a16 sqq.). Par ailleurs, il semble y avoir une équivoque dans la façon dont l'abstraction est convoquée. Si tout processus d'abstraction relève de la pensée ou de la représentation, la réciproque est-elle vraie ? Quand Aristote parle de vónбis en Physique III, il a bien en tête une pensée contrefactuelle (d'où l'interprétation de Simplicius en termes de $\phi \alpha \nu \tau \alpha \sigma i \alpha$, qui semble assez vraisemblable), une « représentation fictive » (p. 57), un « produit spéculatif d'une imagination 
qui marche dans le vide»(p.59), mais est-il légitime de dire que «la faculté d'imagination représentative n'est considérée que comme un pouvoir d'abstraction post rem, sans aucune portée ontologique, puisqu'elle "marche dans le vide" » (p. 47) ? On pourrait au contraire soutenir que Simplicius fait bien la différence entre une représentation résultant d'une abstraction post rem (comme c'est le cas pour les grandeurs mathématiques) et une représentation fictive (imaginaire) qui ne correspond à aucune réalité et que, sur ce point, il est tout à fait en accord avec Aristote. Cette équivoque se retrouve dans les pages consacrées au nombre (p. 66 sqq.), où le nombre monadique est qualifié à plusieurs reprises d'abstrait (après avoir dit que le «nombre monadique est privé d'existence réelle et [que] son infinité est purement imaginaire, voire illusoire », Soulier ajoute quelques lignes plus loin que « le nombre monadique, purement abstrait, n'a pas d'existence réelle, car il est séparé de toute matière et n'a son être que dans la représentation », p. 67), cas dans lesquels l'abstraction semble être comprise dans le sens de «séparation», par opposition à d'autres cas où le terme «abstraction » est utilisé à juste titre (« la grandeur mathématique n'est qu'un accident séparé de son substrat matériel par une opération intellectuelle d'abstraction $\gg$, p. 69).

En quoi consiste donc la « thèse philosophique de Simplicius sur l'ä $\pi \varepsilon ı$ pov » ? D'après Soulier, «la critique de l'illimité imaginaire (...), loin d'épuiser le commentaire de Simplicius, permet au contraire de libérer la place pour une on-

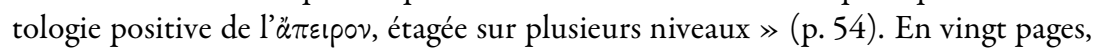
Soulier présente les grandes lignes de l'interprétation de Simplicius : le procès à

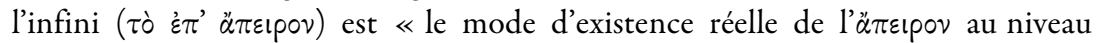
des corps sensibles » (p. 54) et se distingue de l'ä $\pi \varepsilon ı$ pov tout court qui existe seulement dans la représentation, distinction qui, comme le dit l'auteur, recouvre celle entre l'en-puissance et l'en-acte. Ce procès, qui a son être dans le devenir, doit son illimitation à l'illimitation de la matière, laquelle rend possible la division à l'infini des grandeurs et l'addition à l'infini des produits de cette division. « Même si cela peut sembler surprenant dans un horizon néoplatonicien, c'est le lien à la matérialité qui permet de départager entre une illimitation réelle et une illimitation seulement représentée par l'imagination .» (p. 66.)

Dans la deuxième partie («L'ancrage néoplatonicien de la doctrine mobilisée par le Commentaire », p. 75-274), l'auteur expose en détail le contexte néoplatonicien dans lequel Simplicius s'insère. Il commence par présenter la « doctrine néoplatonicienne orthodoxe » concernant la limite et l'illimité en proposant une traduction originale commentée du commentaire de Proclus de la première hypothèse du Parménide (In Parm. 1118.9-1124.37 Cousin), texte dans lequel l'illimité se décline en dix niveaux allant de la puissance infinie de l'Un à l'indétermination de la matière, auxquels correspondent dix niveaux de limite de la limite en soi à la forme engagée dans la matière - (le tout est présenté dans un utile tableau p. 87). L'auteur montre ensuite que Simplicius exploite les quatre 
niveaux inférieurs de l'illimité proclien pour expliquer l'ä $\pi \varepsilon ı$ pov d'Aristote (à savoir, en partant du niveau inférieur, la matière, le corps non qualifié en tant qu'il est divisible à l'infini, les qualités premières et le devenir), et qu'il exploite dans d'autres contextes (notamment dans le commentaire de Physique VIII et le Corollarium de tempore) les acquis du commentaire de Physique III 6, en distinguant deux illimitations du «toujours» (procès à l'infini et infinité simultanée), Simplicius s'appropriant l'héritage proclien tout en critiquant Damascius et Philopon. L'auteur consacre enfin une bonne centaine de pages (p. 161-274) à «l'infinité transcendante » : il montre ici comment le commentaire des passages doxographiques de Physique III est pour Simplicius l'occasion de dégager une conception positive de l'infini. Ainsi, l'arrière-plan nettement néoplatonicien de la réhabilitation opérée par Simplicius des doctrines des pythagoriciens, de Platon et d'Anaxagore fait l'objet d'une étude très méticuleuse : dans chaque cas, l'auteur mesure la distance qui sépare l'exposé aristotélicien du commentaire de Simplicius et comment ce dernier «va jusqu'à déroger à la démarche concordiste qui lui est habituelle » pour mener sa défense d'Anaxagore qui «pourrait presque sembler passionnelle » (p. 208), Anaxagore semblant être «le physicien présocratique qui exprime le mieux les conceptions théologiques qui sont les siennes, celles du néoplatonisme » (p. 209).

La troisième partie (« L'ä $\pi \varepsilon ı$ pov néoplatonicien de Plotin à Damascius », p. 275-448) poursuit l'entreprise de contextualisation du commentaire de Simplicius, mais cette fois en étudiant pour eux-mêmes les développements néoplatoniciens de thématiques ou de problématiques relatives à l'ä $\pi \varepsilon ı$ pov (Simplicius n'est quasiment plus évoqué dans cette partie). Si la partie précédente avait insisté sur la conception néoplatonicienne du double äreıрov (indétermination négative de la matière et infinité positive dans l'intelligible) et sur la façon dont cette conception affleure dans le commentaire de Simplicius, il s'agit ici d'entrer plus avant dans les subtilités de la réflexion néoplatonicienne sur l'infini. Si cette partie étudie les conceptions néoplatoniciennes, elle revient néanmoins en maints endroits, afin de contextualiser ces conceptions elles-mêmes, à l'origine des questions traitées. Ainsi, avant d'aborder Plotin, Soulier s'intéresse aux traitements platoniciens de l'ä $\pi \varepsilon i p o v$ (Philèbe et Timée) et à la difficulté exégétique qui en est issue chez Aristote (doctrines non écrites). De même, dans le long développement qu'il consacre à l'Un et à la dyade indéfinie dans le néoplatonisme, l'auteur en vient, pour présenter l'histoire de la « reconstruction platonicienne du pythagorisme », à retracer, en fait, l'histoire de la dyade depuis le pythagorisme préplatonicien, en passant par l'Ancienne Académie et le médio-platonisme. On retrouve ainsi un sujet épineux déjà abordé dans la deuxième partie, à savoir le statut respectif de la Dyade indéfinie et du Grand et du Petit. Soulier y soutenait (p. 180-206) que Simplicius considère le Grand et le Petit comme principes matériels constitutifs des corps sensibles (illimitation sensible) et la Dyade comme principe constitutif des Idées-Nombres intelligibles (illimitation intelligible) 
(p. 191); il soutient ici que l'invention de la dyade indéfinie ne remonte pas à Platon, et encore moins à l'ancien pythagorisme, mais à Xénocrate (p. 355 sqq.). Remarquons en passant que le témoignage d'Aristote qui est convoqué dans ces différents développements sur la dyade (Métaphysique, A 6, 987b18-22) fait l'objet de traitements différents (les traductions proposées ne sont pas exactement les

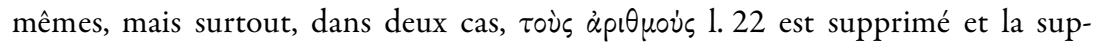
pression est justifiée en notes - p. 182 n. 85 et p. 284 n. 32 -, tandis que lors de

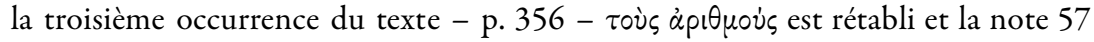
justifie cette conservation sans faire la moindre allusion aux deux citations antérieures). D’une façon générale, le lecteur pourra être gêné par ces développements relatifs à la dyade, qui auraient peut-être gagné à être rassemblés dans une même partie. En revanche, les pages consacrées à Plotin et à Proclus, aussi bien dans cette partie que dans l'ensemble de l'ouvrage, sont tout à fait passionnantes (la lecture des propositions 149-150 des Éléments de Théologie de Proclus est remarquable).

Dans l'assez brève quatrième partie (« La méthode d'exégèse », p. 449-490), qui s'apparente en fait davantage à un appendice, après avoir étudié le okotós d'Aristote selon Simplicius, Soulier s'intéresse à la façon dont il recourt aux commentateurs (Eudème, Alexandre d'Aphrodise, Porphyre, Thémistius) et esquisse une rapide comparaison entre son commentaire et celui de Philopon; enfin, il consacre quelques pages à la façon dont Simplicius reformule les arguments aristotéliciens.

Cet ouvrage, qui tend à tirer tous les fils, essentiellement néoplatoniciens, qui apparaissent de façon plus ou moins explicite dans la trame du commentaire que Simplicius consacre aux chapitres 4 à 8 du livre III de la Physique, ne peut que donner au lecteur l'impatience de lire la nouvelle édition critique du texte de Simplicius avec traduction et notes que l'auteur prépare en collaboration avec P. Golitsis (CUF). 